\title{
40. A Note on the Mean Value of the Zeta and L-functions. VII
}

\author{
By Aleksandar Ivić() and Yoichi MotoHaSHI**) \\ (Communicated by Kunihiko KodAIRA, M. J. A., June 12, 1990)
}

1. Let $E_{2}(T)$ be the error-term in the asymptotic formula for the fourth power mean of the Riemann zeta-function, so that

$$
\int_{0}^{T}\left|\zeta\left(\frac{1}{2}+i t\right)\right|^{4} d t=T P_{4}(\log T)+E_{2}(T)
$$

with a certain polynomial $P_{4}$ of degree 4 . As has been pointed out already in the preceding note [3] of this series, Corollary 2 in it gives an alternative proof of Zavorotnyi's claim [5]:

$$
E_{2}(T)=O\left(T^{(2 / 3)+\varepsilon}\right)
$$

for any fixed $\varepsilon>0$. In fact this is simply a resultant of combining the corollary with the spectral mean of Hecke series ([4]) :

$$
\sum_{\kappa_{j} \leqq x} \alpha_{j} H_{\jmath}\left(\frac{1}{2}\right)^{4} \ll x^{2+\varepsilon} \text {. }
$$

Here $\left\{\lambda_{j}=\kappa_{j}^{2}+(1 / 4), \kappa_{j}>0\right\}$ is the discrete spectrum of the non-Euclidean Laplacian on $S L(2, Z)$, and $\alpha_{j}=\left|\rho_{j}(1)\right|^{2}\left(\cosh \pi \kappa_{j}\right)^{-1}$ with the first Fourier coefficient $\rho_{j}(1)$ of the Maass wave form corresponding to $\lambda_{j}$ to which the Hecke series $H_{j}$ is attached.

Though (2) is the hitherto best result on the upper bound for $E_{2}(T)$ it is generally believed that $T^{(1 / 2)+\varepsilon}$ may be the true order of it. The aim of the present note is to study this problem from the opposite direction. Namely we are going to show that under a hypothesis of the type of nonvanishing theorems for automorphic $L$-functions one may deduce an $\Omega$-result on $E_{2}(T)$ from [Theorem, 3].

To formulate the hypothesis we denote by $\left\{\mu_{h}\right\}$, arranged in the increasing order, the mutually different members in the set $\left\{\kappa_{j}\right\}$. And we put

Now we set out

$$
G_{h}=\sum_{k j=\mu_{h}} \alpha_{j} H_{\jmath}\left(\frac{1}{2}\right)^{3} \text {. }
$$

Hypothesis A. Not all $G_{h}$ vanish.

Then we have

Theorem. Under Hypothesis $A E_{2}(T)=\Omega\left(T^{1 / 2}\right)$ holds.

We note that Zavorotnyi's argument [5] does not seem to be able to yield our theorem. Also the theorem should be compared with the $\Omega$-result on the mean square of $|\zeta((1 / 2)+i t)|$ due to Good [1] (for the latest develop-

* Serbian Academy of Sciences, Beograd, Yugoslavia.

**) Department of Mathematics, College of Science and Technology, Nihon University, Tokyo, Japan. 
ments see Hafner and Ivić [2]).

Remark. (i) If $G_{h}=0$ for all $h$ then the Lindelöf hypothesis for $\zeta(s)$ would follow. (ii) It is clear that Hypothesis $A$ is a consequence of the following stronger statement:

Hypothesis B. There exists $a \lambda_{j}$ of multiplicity one such that $H_{j}(1 / 2)$ $\neq 0$.

Probably this may be checked numerically.

2. We shall give an outline of our proof of the theorem; a detailed version is available in a form of manuscript, and will be published elsewhere.

As in [3] we put

$$
I_{4}(T, \Delta)=(\Delta \sqrt{\pi})^{-1} \int_{-\infty}^{\infty}\left|\zeta\left(\frac{1}{2}+i(T+t)\right)\right|^{4} e^{-(t / \Delta)^{2}} d t,
$$

and consider the expression

$$
\int_{T}^{2 T} I_{4}(t, \Delta) d t=T Q_{4}(\log T)+R(T, \Delta),
$$

where $Q_{4}$ is a polynomial of degree 4 . This is suggested by [Corollary 2, 3], so that $R(T, \Delta)$ is supposed to be of the order of $T^{c}$ with a $c<1$ for some suitably chosen $\Delta$. Then we make the initial observation:

Lemma 1. If $R(T, \Delta)=\Omega(g(T))$ for $\Delta$ satisfying $T^{\varepsilon} \leqq \Delta \leqq T^{1-\varepsilon}$ and $\Delta=$ $o\left(g(T) \log ^{-5} T\right)$ then we have $E_{2}(T)=\Omega(g(T))$.

This can be proved by truncating, in an obvious manner, the double integral involved in (4) and taking (1) into account.

Next we introduce

$$
S(V, \Delta)=\int_{V}^{2 V} R(T, \Delta) d T
$$

If Hypothesis A implies $S(V, \Delta)=\Omega\left(V^{3 / 2}\right)$ for $\Delta$ satisfying $V^{\varepsilon} \leqq \Delta \leqq V^{(1 / 2)-\varepsilon}$ then obviously $R(T, \Delta)=\Omega\left(T^{1 / 2}\right)$ for $\Delta$ satisfying $T^{\varepsilon} \leqq \Delta \leqq T^{(1 / 2)-\varepsilon}$, and Lemma 1 ends the proof of the theorem. Thus we are led to the problem of finding a suitable explicit formula for $S(V, \Delta)$ for $\Delta$ in the indicated range. The reason that we have integrated $R(T, \Delta)$, instead of treating it directly, is that for $S(V, \Delta)$ we can give such an explicit formula but it seems difficult to do so for $R(T, \Delta)$.

In fact, after a somewhat involved computation we have deduced from [Theorem, 3] and (3) the following:

Lemma 2. Uniformly for $V^{\varepsilon} \leqq \Delta \leqq V^{(1 / 4)-\varepsilon}$ we have

$$
\mathrm{S}(V, \Delta)=2 V^{3 / 2} \operatorname{Im}\left\{\sum_{h=1}^{\infty} G_{h}\left(F\left(\mu_{h}\right) V^{i \mu_{h}}+F\left(-\mu_{h}\right) V^{-i \mu_{h}}\right)\right\}+O\left(V^{3 / 2}(\log V)^{-1}\right),
$$

where

$$
F(\mu)=\exp \left(\frac{\pi}{2}\left(\mu+\frac{i}{2}\right)\right)\left((\sinh \pi \mu)^{-1}+i\right) \frac{\Gamma^{3}((1 / 2)-i \mu)\left(2^{(1 / 2)+i \mu}-1\right)\left(2^{(3 / 2)+i \mu}-1\right)}{\Gamma(1-2 i \mu)(1+2 i \mu)(3+2 i \mu)} .
$$

We note that $F(\mu)=O\left(|\mu|^{-5 / 2}\right)$ as $|\mu|$ tends to infinity; this and (3) imply that the last sum over $h$ is absolutely convergent.

Now, providing Hypothesis A, the desired $\Omega$-result for $S(V, \Delta)$ will be 
an easy consequence of the last lemma, once we establish the following general assertion:

Lemma 3. Let $\left\{a_{j}\right\}$ and $\left\{b_{j}\right\}$ be such that $\left|a_{1}\right|>\left|b_{1}\right|$ and $\sum_{j=1}^{\infty}\left(\left|a_{j}\right|+\left|b_{j}\right|\right)<\infty$. Also let $\left\{\omega_{j}\right\}$ be a strictly increasing sequence of positive numbers. Then we have, as $x$ tends to infinity,

$$
\operatorname{Im}\left\{\sum_{j=1}^{\infty}\left(a_{j} x^{i \omega j}+b_{j} x^{-i \omega \jmath}\right)\right\}=\Omega_{ \pm}(1)
$$

To show this we denote the left side by $\varphi_{0}(x)$ and define $\varphi_{n}(x)$ inductively by

$$
\varphi_{n+1}(x)=\int_{x}^{\tau x} \varphi_{n}(x) \frac{d x}{x}
$$

where $\tau=\exp \left(\pi / \omega_{1}\right)$. As is easily seen we have, for any $n \geqq 0$,

$$
\left|2^{-n} \varphi_{n}(x)-\operatorname{Im}\left\{a_{1}\left(\frac{i}{\omega_{1}}\right)^{n} x^{i \omega_{1}}\right\}\right| \leqq\left|b_{1}\right| \omega_{1}^{-n}+\sum_{j=2}^{\infty}\left(\left|a_{j}\right|+\left|b_{j}\right|\right) \omega_{j}^{-n} .
$$

Taking $n$ sufficiently large the right side is less than $\left|a_{1}\right| \omega_{1}^{-n}$, but the member in the braces can be equal to both $i\left|\alpha_{1}\right| \omega_{1}^{-n}$ and $-i\left|\alpha_{1}\right| \omega_{1}^{-n}$ infinitely often. This proves the lemma and thus the theorem.

Remark. Lemma 2 has an obvious counterpart in the theory of prime numbers. That is, in just the same way as the Chebyshev function $\psi(x)$ is related to all complex zeros of $\zeta(s)$ the fourth power moment of $|\zeta((1 / 2)+i t)|$ is related to all eigenvalues of the non-Euclidean Laplacian on $S L(2, Z)$ (or more exactly, all complex zeros of Selberg's zeta-function for $S L(2, Z)$ ).

Addendum. After submitting this paper we learned that A. Good (J. Number Theory, 13, 18-65 (1981)) had obtained an $\Omega$-result of the same strength as ours for Hecke $L$-series associated with holomorphic cusp forms under a certain hypothesis of the type of non-vanishing theorems.

\section{References}

[1] A. Good: Ein $\Omega$-Resultat für das quadratische Mittel der Riemannschen Zetafunktion auf der kritischen Linie. Invent. Math., 41, 233-251 (1977).

[2] J. L. Hafner and A. Ivić: On the mean-square of the Riemann zeta-function on the critical line. J. Number Theory, 32, 151-191 (1989).

[3] Y. Motohashi: A note on the mean value of the zeta and $L$-functions. VI. Proc. Japan Acad., 65A, 273-275 (1989).

[4] L. A. Takhtajan and A. I. Vinogradov: An estimate of the residuum of Rankin's $L$-series. Dokl. Akad. Nauk USSR, 267, 30-34 (1982) (in Russian).

[5] N. I. Zavorotnyi: On the fourth power mean of the zeta-function (preprint) (in Russian). 